\title{
Fusion technique for SAR and Gray Visible Image based on Hidden Markov Model in Non-subsample Shearlet Transform Domain
}

\author{
Jian Liu ${ }^{1, a}$, Yingjie Lei ${ }^{1, b}$, Yaqiong Xing ${ }^{1, c}$, Chuanguo Lu ${ }^{2, d}$ \\ ${ }^{1}$ Air and Missile Defence College, Air Force Engineering University, Xi’an 710051, China \\ ${ }^{2}$ Unit 95806, Beijing 100076, China \\ aliujian758@163.com, 'Plei@ieee.org, 'cffly132@163.com, 'luyujie22@163.com
}

Keywords: Non-subsample Shearlet Transform, Hidden Markov Tree, Image Fusion.

\begin{abstract}
To exact the more directional information and important detail information from the images effectively, a novel image fusion algorithm for SAR and gray visible image based on the Hidden Markov Model in the Non-subsample Shearlet Transform (NSST) domain is proposed. In NSST domain, the low frequency coefficients are fused by standard deviation. Meanwhile, the NHMT model is built to train the high frequency coefficients. After that, the energy of gradient is used to select the trained coefficients. Then, the low frequency and high frequency images are fused by inverse transformation of NSST to get the final image. Finally, the simulation proves that compared with other mufti-scale HMT models and traditional NSST fusion strategy, the proposed method in this paper can promote the fusion quality and enhance the information of the images, reducing noise as well.
\end{abstract}

\section{Introduction}

Image fusion is an important part of information fusion which combines two or more source images obtained from different sensors to a fused one that can be more accurate, clear and reliable. The mufti-scale analysis is widely applied to image fusion because it can represent images sparsely and direction ally. Image fusion method based on Non-sampler Contour let transform (NSCT) is currently a hot research field. However, due to the structure of itself, NSCT is time-consuming, which is unfavorable for the real-time processing. In order to deal with the problems above, NSST is proposed by Beasley, which combined the non-sampler Chaplaincy pyramid transform with several different shearing filters. The implementation of NSST can effectively reduce the Gibbs phenomenon and improve computational efficiency [1], but its applications in image fusion are still being explored [2] [3].

Gray visible images have clearly details, but inability in the condition when the bad weather came or for the all-time observing. The Synthetic Aperture Radar (SAR) is such an important source of information, providing high-resolution images with all weather and illumination conditions. However, due to the inherent presence of speckle, the visibility of the SAR images is limited [4]. In this paper, we will propose an image fusion method based on Hidden Markov Tree in NSST domain (NHMT) for SAR and gray visible image, as the Hidden Markov model can effectively smooth the noises of images [5].

\section{NSST}

The discrete shearlet transform can be obtained by oversampling the shearlet on a proper discrete set. NSST consists of two parts, and the image is first decomposed by the NSP (non-sampler pyramid, NSP), which produces one low-frequency sub-band and $k+1$ high-frequency sub-bands whose sizes are all the same as source image; $k$ denotes the number of decomposition levels. NSP ensures the low-frequency component available alliterative to capture the singularities in the image. After that, the high-frequency sub-bands are decomposed by the shearing filters with $l$ stage at each scale, which will yield $2^{l}$ directional sub-bands with the same size as the source images. In frequency domain, each shearlet is supported on a pair of trapezoids, of approximate size $2^{2 j} \times 2^{j}$, oriented along lines of 
slope $l 2^{-j}$. Fig. 1 shows the proceeding of NSST, and the frequency partition of NSST is show in Fig. 2

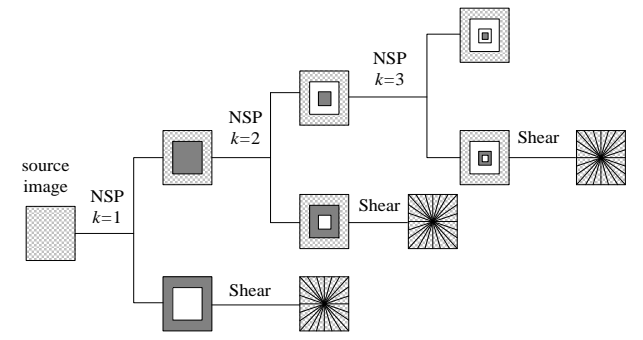

Fig. 1 The multi-scale and multi-directional decompositions of NSST

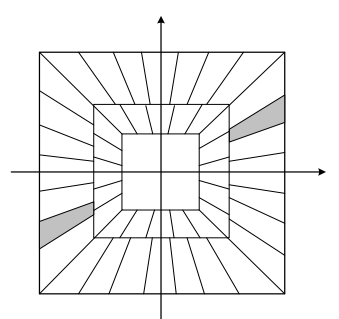

Fig. 2 Frequency partition

\section{The HMT model in NSST domain (NHMT)}

The Hidden Markov tree (HMT) model is derived from the Markov chain, and has a natural parent-child dependency by establishing links between hidden states of the coefficients [6]. Kan earl [7] [8] proposed image fusion based on the Contour let domain HMT model. Because Contour let transform lack of shift-in variance and the coefficients' sparsity is slightly worse than that in NSST domain, and will lead to Gibbs phenomenon. So propose the NSST domain HMT model, in which the transform satisfies the shit-in variance.

We usually use two states Gaussian mixture model (GMM) to describe the probability density function (pd) of the NSST coefficients. The NHMT contains the following parameters at the directional $m_{j}$ in scale $j$ is described as:

$P_{1, k}\left(\mathrm{k}=1,2 \ldots m_{1}\right)$ : The state probability vector of root nodes at each directional sub-band at the coarsest scale.

$A_{j, k}\left(\mathrm{j}=2 \ldots, \mathrm{J} ; \mathrm{k}=1,2 \ldots m_{j}\right)$ : The state transition probability matrix of hidden state from the parent sub-band at scale $\mathrm{j}-1$ to the directional sub-band $k$ at scale $j$.

$\sigma_{j, k}\left(\mathrm{j}=1 \ldots, \mathrm{J} ; \mathrm{k}=1,2 \ldots m_{j}\right)$ : The Gaussian standard deviation vector of the directional sub-band $k$ at scale $j$.

So the parameter vector $\theta=\left\{P_{1, k}, A_{j, k}, \sigma_{j, k}\right\}$ can be obtained. Generally, we usually tie the coefficients within each scale sub-band and let them share the same NHMT parameters. Then the NHMT is trained by the Expectation Maximization (EM) algorithm to estimate the parameters. After the training, we will obtain the pdf $f\left(C_{i}\right)$ of coefficient $C_{i}$, and then get the new coefficient $H_{i}$ by multiplying $C_{i}$ and the normalized $f\left(C_{i}\right)$ as follows:

$$
H_{i}=\frac{f\left(C_{i}\right)}{\left|f\left(C_{i}\right)\right|} \times C_{i}
$$

Fusion of low-frequency coefficients. SD describes the dispersion degree between the gray values and the gray mean values of each pixel. Let $p(i, j)$ denote the gray value of the pixel $(i, j)$, and the size of the image is $M \times N$.Then the SD is calculated as follows:

$$
\begin{aligned}
& \sigma=\sqrt{\frac{1}{M \times N} \sum_{i=1}^{M} \sum_{j=1}^{N}(p(i, j)-\mu)^{2}} \\
& \mu=\frac{1}{M \times N} \sum_{i=0}^{M-1} \sum_{j=0}^{N-1} p(i, j)
\end{aligned}
$$

According to Equations (2) and (3) we calculate the weight value by:

$\omega_{V_{k}^{0}}=s d_{V_{k}^{0}} /\left(s d_{V_{k}^{0}}+s d_{s_{k}^{0}}\right) ; \omega_{s_{k}^{0}}=s d_{s_{k}^{0}} /\left(s d_{V_{k}^{0}}+s d_{s_{k}^{0}}\right)$

So the fused low-frequency sub-band is obtained by:

$F_{\text {low }}=\omega_{V_{k}^{0}} V_{k}^{0}(i, j)+\omega_{S_{k}^{0}} S_{k}^{0}(i, j)$

Where, $s d_{V_{k}^{0}}$ and $s d_{S_{k}^{0}}$ represent the SD of the low-frequency sub-bands. 
Fusion of high-frequency coefficients. As Energy of Gradient (EOG) describes the gradient information of the image and has the ability to represent the definition of the images [9].In this paper, we use the EOG to select high-frequency coefficients, and EOG is defined as follow:

EOG $=\sum_{i=1}^{m} \sum_{j=1}^{n}\left(p_{a}^{2}(i, j)-p_{b}^{2}(i, j)\right)$

Where $p(i, j)$ denotes gray value locate at $(i, j)$,

$p_{a}(i, j)=p(i, j)-p(i-1, j)$

$p_{b}(i, j)=p(i, j)-p(i, j-1)$

Let the size of the local region is $N \times N$, and then the EOG of the local region is:

$\mathrm{EOG}_{L}(i, j)=\sum_{m=-N-1 / 2}^{N-1 / 2} \sum_{n=-N-1 / 2}^{N-1 / 2}\left(p_{a}^{2}(i+m, j+n)-p_{b}^{2}(i+m, j+n)\right)$

According to Equation (8), the selection method of high-frequency coefficients is defined as:

$F_{h i g h}^{l_{k}}(i, j)=\left\{\begin{array}{l}H_{V_{k}^{\prime}}(i, j), \quad \text { if } \operatorname{EOG}_{L}\left(H_{v_{k}^{\prime}}(i, j)\right) \geq \operatorname{EOG}_{L}\left(H_{S_{k}^{\prime}}(i, j)\right) \\ H_{S_{k}^{\prime}}(i, j), \quad \text { if } \operatorname{EOG}_{L}\left(H_{S_{k}^{\prime}}(i, j)\right) \geq \operatorname{EOG}_{L}\left(H_{v_{k}^{\prime}}(i, j)\right)\end{array}\right.$

Where $H_{V_{k}^{\prime}}(i, j)$ and $H_{s_{k}^{\prime}}(i, j)$ represent the new coefficients of high-frequency sub-band trained by NHMT model at direction $k$ at scale $j . F_{\text {high }}^{l_{k}}(i, j)$ is the fusion result at $(i, j)$.

\section{Simulation}

To further demonstrate the effectiveness of the proposed method (M4), three algorithms for comparison: (1): HMT model in wavelet domain (M1) [10]; (2): HMT model in Contour let domain (M2) [11]; (3): The traditional image fusion rules based on NSST (M3).

It is worth noting that all the images are all decomposed in to 3 levels by Wavelet, Contour let and NSST. The pyramid filter for Wavelet is set as 'db8'. The pyramid filter and directional filter for Contour let is set as '9-7pkva'.Then the decomposition level of Contour let is set as [1,2,3].For the proposed method, the pyramid filter of NSST is set as 'flat mate and directional filter is set as 'shear'. As there is no restriction on number of directions decomposed by NSST, the number of the directions of NSST is set as [ 14 16]. In addition, the convergence precision of EM algorithm used to estimate the parameters of NHMT is within $10^{-4}$. In this paper, we chose SD, Mutual Information (MI) and Peak Signal to Noise Ratio (PSNR) as objective evaluation criteria for comparing the performance of each methods.

Fig. 4 shows that all of the four methods are able to extract the useful information from the source image and produce the fused image $\mathrm{F}$, but there still exit some differences in the quality of the fused images. The information of the big building in the center of the fused image based on M1 and M3 do not acquire sufficient descriptions compared with those based on M2 and M4.This is because the fusion result of M3 is suffered from the speckle noise. Though M1 can suppress the noise in some extent, the contrast levels of the fused image based on M1 is remarkably inferior to those based on others. This may because the drawback of Wavelet in presenting mufti-scale and mufti-directional. Obviously, the fused image concerning M2 and M4 is of higher definition and more details in buildings and roads. Compared with M2, M4 not only have a reasonable contrast level, but also absorb more detail information of backgrounds from the source images.

Table 1 comparison of the fusion methods

\begin{tabular}{cccc}
\hline METHOD & $S D$ & MI & PSNR \\
\hline M1 & 38.5388 & 3.3663 & 17.8062 \\
M2 & 38.6363 & 3.3258 & 21.7465 \\
M3 & 40.9197 & 2.9844 & 16.6239 \\
M4 & 45.5080 & 4.2035 & 24.7949 \\
\hline
\end{tabular}




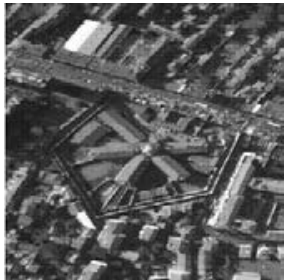

(a) image $\mathrm{V}$

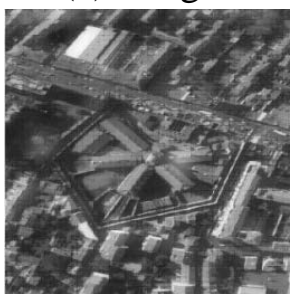

(d) result based on $\mathrm{M} 2$

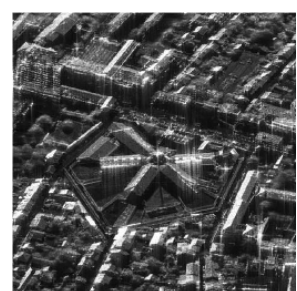

(b)SAR

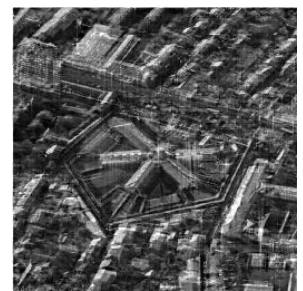

(e) result based on $\mathrm{M} 3$

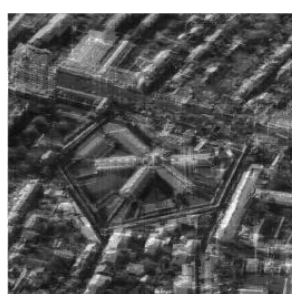

(c)result based on M1

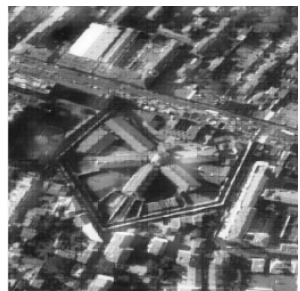

(f) result based on M4

Fig.3 Fused image based on M1-M4

The results of objective evaluation criteria based on M1-M4 are listed in Tab.1. Tab.1 shows that M3 has lower value in MI and PSNR, but better performance in SD. M1 has a good performance in MI, and M3 has a good performance in PSNR. In addition, it also indicates that M4 are much better than other methods on the whole in terms of objective result. For the M4, the SD and PSNR are about $11 \%$ higher than those from the others, and the value of MI reaches 4 . The above data shows that the fused image based on M4 can effectively remove noise in SAR image and extract important details information from source images.M4 has excellent performance both in qualitative and quantitative analysis, and thereby can improve application value of SAR and gray visible images in other fields.

\section{Conclusions}

In this paper, a new image fusion method based on HMT model in NSST domain is introduced. According to the characteristics of low-frequency sub-band and high-frequency sub-band, low-frequency coefficients are fused by using SD rules. For high-frequency coefficients, dependencies are established across scales, and the new coefficients are trained by the NHMT model. We combine the new coefficient with the EOG to select the high-frequency coefficients. Simulation results show that the proposed method for SAR and gray visible image not only makes better performance in expressing the edges, textures and structural features of grey visible image but also reduce the speckle noise in SAR images, enhancing the fused result of SAR and gray visible image. The algorithm is considered of great use in scientific research and engineering practice.

\section{References}

[1] S. Cheng. Research on Image Fusion Algorithm Based on Shearlet [D]. Xian: Dianne University, 2012.

[2] L. J. Liang, L. Lin, Z. S.Lin. Nonscheduled Shearlet-Based Image Denouncing Using Multistage Products[C].ICICIP, Beijing, China.2013:p. 476-480.

[3] H. Biao, Z. Xiaohua, B. Grooming, F. Hongxiao. SAR Image Despeckling Based on Nonsubsampled Shearlet Transform [J].IEEE Journal of Selected Topics in Applied Earth Observations and Remote Sensing, 2012, 5(3):p. 809-823.

[4] J. H. Yan, J. L.Chen, L. Fang. SAR Image Denoising Based on Curvelet Domain Hidden Markov Tree Models [J]. Chinese Journal of Computers, 2007, 30(3):p. 492-497.

[5] W. Yan, Z. Peng, L. Ming. SAR image multitasks segmentation using a multistage and bidirectional triplet Markov fieds model in nonscheduled contour let transform domain [J].Information Fusion, 2013, 14:p. 441-449. 
[6] C. H. Sheng, W.J. Tsai. A framework for video event classification by modeling temporal context of multimedia features using HMM [J].J. Vis. Commun. Image R. 2014, 25: p. 285-295.

[7] L. Kun. G. Lei, C.J. Song. Image Fusion Algorithm Based on Contourlet Domain Hidden Markov Tree Model. Acta Photonica Sinica, 2010, 39(8):p. 1384-1387.

[8] L. Z. Wen, J. L. Cheng, J. H. Yan. Image fusion algorithm using the Contourlet HMT model. Journal of XiDi an University, 2008, 35(3):p. 434-438.

[9] Q.H. Lin, Z.H. Xin, L.Q. Chang, L. Rui. Suppression of infrared image background by multiscale hidden Markov model. Optics and Precision Engineering, 2011, 19(8): p. 1951-1955.

[10]L.H. Feng. Research on Pixel Level Fusion Methods for Multifocus Image [D]. Chongqing: Chongqing University, 2012. Signal Processing, 1998, 46(4): p. 1053-105.

[11] D. Lei,J. W. Guo,C.Y. Hao. Remote Sensing Image Fusion Method Based on Contourlet Domain Hidden Markov Tree Model [J]. J.Infrared Millmi. Waves, 2008, 27(4): p. 286-289. 